\title{
Estratégias de coping e sintomas de stress na adolescência
}

\section{Coping strategies and stress symptoms in adolescence}

\author{
Christian Haag KRISTENSEN ${ }^{1}$ \\ Luiziana Souto SCHAEFER' \\ Fernanda de Bastani BUSNELLO'
}

\begin{abstract}
Resumo
As estratégias de coping são orientadas para a redução do stress, envolvendo reações físicas ou emocionais. O objetivo geral deste estudo foi identificar estratégias de coping utilizadas por adolescentes diante de eventos estressores, bem como a manifestação de sintomas de stress. Participaram da pesquisa 220 adolescentes, de ambos os sexos, provenientes de uma escola da rede pública de Porto Alegre (RS). Os instrumentos utilizados foram o Inventário de Estratégias de Coping e a Escala de Stress Infantil. Os resultados demonstraram que as estratégias mais utilizadas foram autocontrole, afastamento e fuga e esquiva. Entre os indivíduos que apresentaram mais sintomas de stress, as estratégias mais utilizadas foram fuga e esquiva, suporte social, confronto e afastamento.
\end{abstract}

Unitermos: Adolescência. Estratégias de enfrentamento. Stress.

\begin{abstract}
Coping strategies are directed towards the reduction of stress, involving physical or emotional reactions. The overall aim of this study was to identify coping strategies among adolescents exposed to stressful events. Two-hundred and twenty adolescents, ages of 12 and 18 years, male and female, studying in a public school in Porto Alegre (RS), took part of this research. The instruments used were the Coping Strategies Inventory, the Child Stress Scale and school grades. It was found that the coping strategies most frequently employed were Self-control, Withdrawal and Escape-Avoidance. Amongst adolescents with the greatest number ofstress-related symptoms, the most frequent coping strategies were Escape-Avoidance, Social Support, Confrontation and Withdrawal.
\end{abstract}

Uniterms: Adolescence. Coping strategies. Stress.

Estratégias de coping são recursos cognitivos, emocionais e comportamentais que o indivíduo emprega na tentativa de lidar com situações estressoras (Lisboa et al., 2002). O stress está associado a diversas mudanças no funcionamento do organismo, incluindo alterações tanto em sistemas físicos (como sistema endócrino, sistema imune e sistema nervoso), quanto em sistemas comportamentais, emocionais e cognitivos. Muitas dessas alterações acabam por agravar a desadaptação do indivíduo ao ambiente de desenvolvimento (Gazzaniga \& Heatherton, 2005), sobretudo no ambiente escolar.

Sintomas de stress não aparecem somente em indivíduos adultos. Na infância, diversas situações

$\cos$

1 Pontifícia Universidade Católica do Rio Grande do Sul, Programa de Pós-Graduação em Psicologia. Av. I piranga, 6681, Prédio 11, Sala 933, 90619-900, Porto Alegre, RS, Brasil. Correspondência para/Correspondence to: C. H. KRISTENSEN. E-mail: <christian.kristensen@pucrs.br>. 
estressantes podem ocorrer, incluindo aquelas associadas à dinâmica familiar, como o relacionamento conjugal dos pais, violência doméstica, agressão verbal e morte na família (Kristensen, Leon, D'Incao \& Dell'Aglio, 2004; Romano, 1997; Ryan-Wenger, Sharrer \& Campbell, 2005; Taxis, Rew, Jackson \& Kouzekanani, 2004). Durante os anos da adolescência, o stress pode se manifestar tanto a partir das modificações sociais e cognitivas que o indivíduo vivencia nesse período quanto como consequência das alterações biológicas que caracterizam a puberdade (Lipp, 1989). Conforme a Organização Mundial da Saúde (2005), a adolescência é o período da vida que compreende a faixa etária entre os 10 e 19 anos. No Brasil, segundo o Estatuto da Criança e do Adolescente (2007) - Lei no 8.069, de 13 de julho de 1990, é considerado adolescente o indivíduo que tem entre 12 e 18 anos.

Apesar de a maior parte dos trabalhos sobre coping em crianças e adolescentes basear-se na teoria de stress de Lazarus e Folkman (1984), é importante que as estratégias, recursos e estilos de coping desses indivíduos sejam estudados levando-se em conta suas características biológicas e psicológicas específicas, bem como sua dependência em relação ao adulto (Antoniazzi, Dell'Aglio \& Bandeira, 1998). Nesse sentido, Ryan-Wenger (1992) ressalta que uma teoria de stress-coping específica para este público é necessária, tendo em vista que os estressores nessa etapa da vida não são os mesmos da fase adulta.

\section{Stress e estratégias de coping}

O stress se desenvolve quando as tensões do dia a dia mostram-se superiores à capacidade do indivíduo de dominá-las e/ou superá-las, impossibilitando-o de resistir e de criar estratégias para lidar com elas. O stress altera a qualidade de vida da pessoa, causando diminuição da motivação necessária para as atividades diárias, especialmente nos desafios que o indivíduo encontra cotidianamente. Além disso, provoca a sensação de incompetência, com consequente queda da autoestima (Lipp \& Novaes, 2000).

Diferentes variáveis têm sido associadas pela literatura aos sintomas de stress. Dentre os aspectos mais apontados, destacam-se: traços de personalidade, baixa 22 capacidade de resiliência, falta de redes de apoio, aspectos socioeconômicos e aspectos familiares, como falta de coesão familiar e elevada presença de afetividade negativa (Gazzaniga \& Heatherton, 2005; Gerrig \& Zimbardo, 2005). Estratégias de coping também têm sido relacionadas a situações estressoras e ao desencadeamento do stress (Compas, Malcarne \& Fondacaro, 1988; Dell'Aglio \& Hutz, 2002; Raimundo \& Pinto, 2006).

Não existe uma tradução para a língua portuguesa da palavra coping, apesar de muitos autores utilizarem a palavra enfrentamento. Pode-se definir coping como estratégias utilizadas de forma consciente para lidar com o stress percebido (Lisboa et al., 2002). As estratégias de coping são orientadas para a redução do stress, envolvendo reações físicas ou emocionais (Antoniazzi et al., 1998). Dell'Aglio e Hutz (2002) apontam que a teoria de stress de Lazarus e Folkman (1984) é a que mais tem sido utilizada nos estudos sobre processos de coping, definindo-o como um conjunto de esforços, cognitivos e comportamentais, que os indivíduos utilizam para lidar com situações que avaliam como estressoras.

Segundo o modelo de Folkman e Lazarus (1980), o processo de coping envolve quatro características principais: a) interação do indivíduo com o ambiente; b) administração da situação estressora, em vez de controle ou domínio da mesma; c) avaliação da situação, ou seja, como o fenômeno é percebido, interpretado e cognitivamente representado na vida do indivíduo; d) mobilização de esforços, através dos quais os indivíduos irão empreender ações cognitivas e comportamentais para administrar as demandas internas ou externas que surgem da sua interação com o ambiente. Lazarus e Folkman (1984) propõem oito fatores para o estudo do coping: confronto (esforços agressivos de alteração da situação, podendo apresentar grau de hostilidade e risco envolvido); afastamento (esforços cognitivos de desprendimento e minimização da situação); autocontrole (esforços de regulação dos próprios sentimentos e ações); suporte social (procura de suporte informativo, suporte tangível e suporte emocional); aceitação de responsabilidade (reconhecimento do próprio papel na situação e tentativa de recompor o problema); fuga e esquiva (estratégias para escapar ou evitar o problema); resolução de problemas (esforços focados sobre o problema, buscando alterar a situação); reavaliação positiva (criação de significados positivos, podendo, também, apresentar dimensão religiosa). 
Há controvérsias entre os pesquisadores quanto a considerar o coping como um processo disposicional - mais relacionado a características de personalidade do indivíduo - ou como um processo situacional - entendido como um processo cognitivo modificado em função do tempo e da situação estressante (Dell'Aglio, 2003). Os resultados do estudo feito por Dell'Aglio e Hutz (2002) permitiram verificar que as estratégias de coping não são simplesmente disposicionais, mas determinadas pelo contexto do evento estressante.

Entretanto, parece haver um consenso entre os autores em relação à importância de se distinguir dois tipos fundamentais de coping: um focalizado no problema e outro focalizado na emoção, sendo que a escolha por um deles depende de uma avaliação da situação estressora na qual o indivíduo encontra-se envolvido (Dell'Aglio, 2003). Folkman e Lazarus (1980), a partir de uma perspectiva cognitivista, definem o coping focalizado no problema como o esforço despendido pelo sujeito para mudar ou administrar alguns aspectos de um ambiente, uma pessoa ou uma relação percebida como estressante, enquanto o coping focalizado na emoção envolve esforços para administrar ou regular as emoções negativas associadas ao episódio de stress (Dell'Aglio, 2003). Antoniazzi et al. (1998) apontam que, no final da década de 1990, foi apresentada uma terceira estratégia de coping, focalizada nas relações interpessoais, ou seja, para enfrentar a situação estressante, o indivíduo busca apoio de pessoas próximas.

Também é importante destacar que diversos pesquisadores distinguem "estilos de coping" e "estratégias de coping", pois, embora o primeiro possa influenciar o segundo, são fenômenos independentes e têm diferentes origens teóricas (Antoniazzi et al., 1998; Ryan-Wenger, 1992). Os estilos de coping referem-se a características de personalidade, ou seja, às formas habituais que a pessoa utiliza para lidar com o stress percebido (Antoniazzi et al., 1998). Já o termo estratégias de coping diz respeito a comportamentos ou ações cognitivas tomadas no curso de um episódio particular de stress, isto é, as estratégias de coping estão relacionadas a fatores situacionais, podendo mudar de momento para momento durante os estágios da situação de stress (Antoniazzi et al., 1998). Assim, o foco deste estudo foi as estratégias de coping utilizadas por adolescentes expostos a eventos estressores.

\section{Coping em crianças e adolescentes}

No estudo sobre estratégias de coping em crianças e adolescentes, é necessário compreender seu contexto social, já que ambos dependem do adulto para sua sobrevivência (Compas, 1987). Além disso, Peterson (1989) destaca a importância de se considerar o nível de desenvolvimento cognitivo, visto que este irá influenciar a maneira como a criança ou o adolescente avaliam a situação estressante e, consequentemente, as estratégias que irão utilizar para lidar com tal situação. Piko (2001) também enfatiza que devem ser considerados os fatores demográficos, pessoais, socioculturais e ambientais, entre outros. Dessa maneira, ao compreender o processo de coping como uma interação do indivíduo com seu ambiente, deve-se considerar que as estratégias variam de acordo com os contextos nos quais a pessoa interage, bem como de acordo com suas características pessoais (Lisboa et al., 2002). Ryan-Wenger (1992) aponta que os estressores das crianças são diferentes dos estressores dos adultos, pois aqueles se referem a situações com membros da família, professores, amigos ou a condições socioeconômicas que estão fora do seu controle direto. Por último, os fatores situacionais também influenciam as respostas de coping e determinam mudanças nos tipos de estratégias utilizadas (Lisboa et al., 2002).

Adolescência deriva da palavra latina Adolecere, que significa crescer, desenvolver-se, tornar-se jovem (Becker, 1994). É um período marcado por intensas mudanças físicas, cognitivas e psicossociais, e é considerada uma fase de transição entre a infância e a idade adulta (Becker, 1994; Castro, 1998; Kaplan, Sadock\& Grebb, 1997; Levi \& Schimitt, 1996; Osório, 1992; Outeiral, 1994; Palacios, 1995). Kaplan et al. (1997) também caracterizam a adolescência como um período preparatório para a vida adulta.

Dessa maneira, Câmara e Carlotto (2007) apontam que esta etapa da vida é um momento de fragilidade, especialmente em um contexto sociocultural que determina os papéis apropriados para cada gênero, não somente no sentido de definição, mas também no de adequação massiva a normas e padrões comportamentais. Além disso, os autores ressaltam que, durante esse período, os adolescentes estão testando suas possibilidades e sua capacidade de lidar com os problemas 
que surgem. Os eventos estressores vivenciados nesta fase podem influenciar, também, o processo de aprendizagem, impedindo o sujeito de obter um bom desempenho em suas atividades e gerando uma série de dificuldades neste processo (Kaefer, 2006). O ato de aprender pode sofrer impacto de fatores relacionados à escola (condições físicas da sala de aula, condições pedagógicas, material didático, relacionamento com o professor) e à família (escolaridade dos pais, importância dada pelos pais à aprendizagem, condições socioeconômicas da família, problemas de drogadição, alcoolismo, desemprego) (Antoniazzi et al., 1998; Byrne, Compas, 1987; Davenport \& Mazanov, 2007). Além disso, aspectos da própria pessoa podem interferir no desempenho escolar, como problemas físicos, psicológicos ou neurológicos. Em consequência disto, o próprio fracasso escolar pode se caracterizar como mais um estressor para a vida do indivíduo. Assim, o estudante pode experimentar sentimentos de insegurança, timidez, ansiedade, baixa autoestima e falta de motivação (Rotta, 2006). Diante de todos esses estressores típicos do período da adolescência e do seu impacto para o desenvolvimento dos indivíduos, é fundamental compreender como os adolescentes enfrentam as dificuldades encontradas nessa etapa da vida. Assim, as estratégias de coping que os indivíduos utilizam para se adaptar a situações adversas interferem não somente na saúde mental, mas também na saúde física, no bem-estar social e, consequentemente, na aprendizagem.

Ryan-Wenger (1992) identificou 145 estratégias de coping utilizadas por crianças em situações estressantes, o que possibilitou identificar 15 categorias de coping: atividades agressivas, comportamento de evitação, comportamento de distração, distração cognitiva, evitação cognitiva, reestruturação cognitiva, solução cognitiva de problemas, expressão emocional, resistência, busca de informações, atividades de isolamento, atividades de autocontrole, busca de suporte social, busca de suporte espiritual e modificação do estressor.

Em um estudo feito por Lisboa et al. (2002), os resultados permitiram verificar que a estratégia de coping mais utilizada pelas crianças com idade entre 7 e 12 anos, quando enfrentam problemas com seus colegas, é a busca do apoio de alguém (pais, irmãos mais velhos, professores etc.). Entretanto, ao lidar com problemas com suas professoras, a estratégia mais relatada pelas crianças foi "não fazer nada". A partir desses achados, pode-se compreender o processo de coping mais como situacional do que disposicional, enfatizando mais as características da situação estressante do que as características de personalidade do indivíduo.

Dell'Aglio e Hutz (2002) fizeram um estudo que investigou estratégias de coping em 215 crianças e adolescentes, de 7 a 15 anos, de ambos os sexos, que frequentavam escolas públicas da periferia. Os resultados demonstraram que as crianças de 7 a 10 anos utilizaram mais as estratégias de inação e busca de apoio, e os adolescentes de 11 a 15 anos utilizaram mais a estratégia de ação direta. Quando o evento estressor envolvia pessoas da mesma idade, foram mais utilizadas estratégias de ação agressiva e busca de apoio, enquanto nos eventos com adultos as estratégias mais utilizadas foram aceitação, evitação e expressão emocional. A partir dos resultados desse estudo, os autores concluíram que o coping pode ser compreendido como um processo situacional.

Diversas pesquisas apontam diferenças em relação ao sexo e à idade no uso das estratégias de coping. Dell'Aglio (2003) aponta que as diferenças referentes ao sexo podem ser explicadas pelas diferentes formas de socialização entre meninas e meninos, destacando que os meninos tendem a utilizar estratégias de coping competitivas e as meninas estratégias pró-sociais. Além disso, a utilização de determinadas estratégias de coping também está relacionada às expectativas de papel de cada gênero perante a sociedade. Câmara e Carlotto (2007) destacam que, no Brasil, ainda se atribui ao homem a razão, o controle e a liberdade e, à mulher, uma postura mais passiva e conformista. Assim, a questão de gênero decorre tanto de um modelo de transmissão familiar quanto de transmissão social (Bussey \& Bandura, 1999; Câmara \& Carlotto, 2007).

Quanto à idade, Compas et al. (1991) afirmam que as habilidades para coping focalizadas no problema parecem ser adquiridas mais cedo e as habilidades de coping focalizadas na emoção tendem a aparecer na infância tardia, desenvolvendo-se durante a adolescência, visto que as crianças muito pequenas não conseguem ter consciência de seus próprios processos emocionais e autorregular suas emoções (Antoniazzi et al., 1998). A partir de um estudo feito por Losoya, Eisenberg e Fabes (1998), verificou-se que, com a idade, as crianças 
passam a usar com mais frequência estratégias que requerem um processo cognitivo mais elaborado e se tornam mais independentes, buscando menos o apoio de outras pessoas para lidar com situações estressoras.

Um estudo feito por Câmara e Carlotto (2007), com 389 adolescentes estudantes do terceiro ano do ensino médio de diferentes escolas de Porto Alegre, procurou verificar a associação entre bem-estar psicológico e estratégias de coping nos sexos masculino e feminino. Os resultados mostraram que as estratégias de aproximação do problema, tanto em nível cognitivo como comportamental, contribuem para um índice mais elevado de bem-estar psicológico, tanto para meninas quanto para meninos. A pesquisa também revelou que as meninas apresentam um perfil mais autodirecionado, enquanto os meninos têm um perfil mais voltado para a busca de apoio externo. No entanto, no estudo feito por Dell'Aglio e Hutz (2002), com 55 crianças com idade entre 8 e 10 anos, não foram observadas diferenças entre os sexos quanto às estratégias de coping utilizadas. Portanto, destaca-se a importância de que sejam realizados mais estudos que permitam esclarecer se há diferença entre os sexos quanto à utilização de estratégias de coping. Assim, considerando a importância de se compreender como os adolescentes enfrentam as situações de conflito, o objetivo deste estudo foi identificar estratégias de coping utilizadas por adolescentes diante de eventos estressores, bem como a manifestação de sintomas de stress entre os participantes.

\section{Método}

\section{Participantes}

A amostra foi não probabilística, por conveniência, constituída de 220 estudantes adolescentes com idade entre 12 e 18 anos (Média - $M=15,13$; Desvio-Padrão - DP = 1,5), de ambos os sexos, compreendidos entre a 7 a série do ensino fundamental e o $2^{\circ}$ ano do ensino médio, provenientes de uma escola da rede pública de Porto Alegre (RS). Foram excluídos da amostra os participantes que não concordaram em participar da pesquisa, bem como alunos com idade inferior a 12 anos ou superior a 18 anos. Quanto à distribuição por gênero, 40,7\% eram do sexo masculino e 59,3\% do sexo feminino. Quanto à distribuição por série, 26,2\% pertenciam à 7 a série do ensino fundamental, 25,8\% à $8^{a}$ série do ensino fundamental, 21,3\% ao $1{ }^{\circ}$ ano do ensino médio e 26,7\% ao 20 ano do ensino médio.

\section{Instrumentos}

Foram utilizados para a coleta dos dados os seguintes instrumentos:

- Inventário de Estratégias de Coping (Lazarus \& Folkman, 1984): utilizado para avaliar como os adolescentes lidam com as demandas internas ou externas de um evento estressante. É composto por 66 itens que incluem pensamentos e ações, e cuja intensidade é medida por meio de uma escala de 0 (não utiliza) a 3 pontos (utiliza em grande quantidade). Os itens que compõem o inventário são divididos em oito fatores classificatórios, que foram reorganizados e mantidos por Savóia, Santana e Mejias (1996) após a verificação da confiabilidade e validade à realidade brasileira. São eles: confronto (itens 7, 17, 28, 34, 40, 47), afastamento (itens 6 , $10,13,16,21,41,44)$, autocontrole (itens 14, 15, 35, 43, 54), suporte social (itens $8,18,22,31,42,45$ ), aceitação de responsabilidade (itens 9, 25, 29, 48, 51, 52, 62), fuga e esquiva (itens 58,59), resolução de problemas (itens 1 , $26,46,49$ ) e reavaliação positiva (itens $20,23,30,36,38$, $39,56,60,63)$.

- Escala de Stress Infantil (ESI) (Lipp \& Lucarelli, 1998): tem por objetivo verificar a existência ou não de stress. Consiste de 35 itens agrupados em quatro fatores, correspondentes às respostas de stress: reações físicas, reações psicológicas, reações psicológicas com componente depressivo e reações psicofisiológicas. Ao lado de cada item do instrumento, há um círculo dividido em quatro partes, que devem ser pintadas de acordo com a intensidade do sintoma percebido pela criança: se o sintoma nunca acontece, o círculo fica em branco; se acontece um pouco, uma parte é pintada; se acontece às vezes, duas partes são pintadas; se acontece quase sempre, três partes são pintadas; para sintomas que ocorrem sempre, todo o círculo deve ser pintado.

\section{Procedimentos}

Esta pesquisa foi aprovada pelo Comitê de Ética da Faculdade de Psicologia da Pontifícia Universidade 
Católica do Rio Grande do Sul (Processo 08/04100). Foram realizados, inicialmente, contatos com a escola, a fim de obter autorização para a realização da pesquisa entre seus alunos, por meio de uma carta de apresentação e de autorização; posteriormente, foi estabelecido contato com as turmas a serem avaliadas. Foi enviado aos alunos o Termo de Consentimento Livre e Esclarecido e, aos responsáveis pelos alunos menores de idade, o Termo de Autorização da Participação, explicitando os objetivos da pesquisa e os procedimentos que seriam realizados para o estudo.

Esse estudo teve natureza descritiva, com dados quantitativos, em um delineamento ex-post-facto, do tipo correlacional. A aplicação da ESI e do Inventário de Estratégias de Coping ocorreu em sala de aula, sendo utilizado apenas um período de aula para o preenchimento dos dois instrumentos, com duração média de 30 minutos. Os resultados da ESI e do Inventário de Estratégias de Coping foram levantados e tabulados em banco de dados. A análise descritiva envolveu medidas de tendência central, dispersão e propriedades da distribuição para as variáveis de interesse. A análise inferencial envolveu, primariamente, medidas correlacionais entre as variáveis de interesse (sintomas de stress e estratégias de coping). A comparação entre grupos ocorreu por meio de teste de diferença de média para amostras não pareadas. Todas as análises foram conduzidas no programa SPSS for Windows (Versão 13.0).

O Inventário de Estratégias de Coping avalia a frequência de utilização de cada estratégia (não usa, usa um pouco, usa bastante e usa em grande quantidade). Portanto, para a análise das estratégias de coping mais utilizadas, considerou-se, apenas, se o estudante usou ou não usou cada estratégia. Assim, as estratégias de coping mais utilizadas foram: autocontrole (95,9\%), afastamento (93,7\%), fuga e esquiva (93,5\%), suporte social (92,7\%), reavaliação positiva (92,3\%), resolução de problemas $(91,9 \%)$ e aceitação de responsabilidade (91,9\%). As estratégias de confronto foram as menos utilizadas (85,5\%). A Tabela 1 apresenta a frequência de utilização para cada estratégia. Nesta tabela, é possível visualizar que apesar de a estratégia de autocontrole ter sido utilizada por um maior número de participantes, a estratégia de fuga e esquiva foi empregada com mais

26 frequência.
Tabela 1. Frequência de utilização das estratégias de coping. Porto Alegre (RS), 2008

\begin{tabular}{lcccc}
\hline Fatores & $\begin{array}{c}\text { Não } \\
\text { usou }\end{array}$ & Pouco & Bastante & $\begin{array}{c}\text { Grande } \\
\text { quantidade }\end{array}$ \\
\hline 1. Confronto & 14,5 & 56,5 & 26,3 & 2,7 \\
2. Afastamento & 6,3 & 65,2 & 27,6 & 0,9 \\
3. Autocontrole & 4,1 & 47,9 & 46,6 & 1,4 \\
4. Suporte social & 7,3 & 40,9 & 47,3 & 4,5 \\
5. Aceitação de & 8,1 & 39,4 & 48,4 & 4,1 \\
$\quad$ responsabilidade & & & & \\
6. Fuga e esquiva & 6,5 & 21,1 & 35,1 & 37,3 \\
7. Resolução de & 8,1 & 36,2 & 45,7 & 10,0 \\
$\quad$ problemas & & & & 4,1 \\
8. Reavaliação positiva & 7,7 & 47,1 & 41,1 & \\
\hline
\end{tabular}

Nota. Valores expressos em porcentagens.

De um modo geral, dentre as estratégias de coping mais apontadas pelos adolescentes do sexo masculino é possível citar, em ordem decrescente: resolução de problemas $(M=1,62)$, aceitação de responsabilidade $(M=1,60)$, autocontrole $(M=1,49)$ e afastamento $(M=1,32)$. As meninas, por sua vez, assinalaram mais as seguintes estratégias: fuga e esquiva $(M=1,87)$, suporte social $(M=1,47)$, reavaliação positiva $(M=1,43)$ e confronto $(M=1,16)$. Quando a diferença por sexo foi analisada, verificou-se significância estatística apenas na utilização de estratégias de afastamento [t(219)=2,04; $p=0,042]$. Adicionalmente, verificou-se tendência à significância estatística na diferença por sexo quanto à utilização da estratégia de aceitação de responsabilidade $[t(219)=1,83$, $p=0,069]$ (Tabela 2).

Ao correlacionar as estratégias de coping com os resultados obtidos na ESI, verificou-se que os indivíduos que apresentaram mais sintomas de stress utilizaram significativamente mais estratégias de fuga e esquiva $(M=2,25)$, suporte social $(M=1,62)$, confronto $(M=1,51)$ e afastamento $(M=1,32)$. Além disso, essas estratégias foram utilizadas mais frequentemente por esse grupo, se comparado aos sujeitos com menos sintomas de stress. A comparação também evidencia que estes, por sua vez, utilizaram mais estratégias de autocontrole $(M=1,42)$ e resolução de problemas $(M=1,40)$.

\section{Discussão}

As estratégias de coping mais utilizadas pelos adolescentes foram autocontrole, afastamento e fuga e 
Tabela 2. Utilização de estratégias de coping entre os sexos. Porto Alegre (RS), 2008.

\begin{tabular}{|c|c|c|c|c|c|c|}
\hline \multirow{3}{*}{ Estratégia } & \multicolumn{6}{|c|}{ Sexo } \\
\hline & \multicolumn{2}{|c|}{ Masculino } & \multicolumn{2}{|c|}{ Feminino } & \multicolumn{2}{|c|}{ Total } \\
\hline & M & DP & M & DP & M & DP \\
\hline 1. Confronto & 1,05 & 0,63 & 1,16 & 0,63 & 1,12 & 0,63 \\
\hline 2. Afastamento & 1,32 & 0,53 & 1,17 & 0,51 & 1,23 & 0,52 \\
\hline 3. Autocontrole & 1,49 & 0,56 & 1,44 & 0,55 & 1,46 & 0,56 \\
\hline 4. Suporte social & 1,34 & 0,68 & 1,47 & 0,62 & 1,42 & 0,65 \\
\hline 5. Aceitação de responsabilidade & 1,60 & 0,61 & 1,44 & 0,63 & 1,50 & 0,63 \\
\hline 6. Fuga e esquiva & 1,75 & 0,92 & 1,87 & 0,95 & 1,82 & 0,94 \\
\hline 7. Resolução de problemas & 1,62 & 0,73 & 1,30 & 0,65 & 1,46 & 0,68 \\
\hline 8. Reavaliação positiva & 1,39 & 0,62 & 1,43 & 0,60 & 1,41 & 0,61 \\
\hline
\end{tabular}

M: média; DP: desvio-padrão.

esquiva, e as menos utilizadas foram as estratégias de confronto. Esses resultados não corroboram os achados de Dell'Aglio e Hutz (2002), que apontaram que indivíduos de 7 a 10 anos utilizaram mais as estratégias de inação e busca de apoio, enquanto o grupo de 11 a 15 anos utilizou mais a estratégia de ação direta. Essa discordância entre os dados pode ser explicada pelo fato de que o estudo aqui descrito avaliou as estratégias de coping utilizadas por adolescentes sem considerar eventos estressores específicos, enquanto estudos anteriores identificaram estratégias situacionais, ou seja, esforços empreendidos pelos indivíduos diante de um evento estressor particular, como distúrbios digestivos, depressão, câncer, violência doméstica, psoríase, stress ocupacional e abuso sexual (Calsbeek, Rijken, Bekkers, Henegouwen \& Dekker, 2006; Calvete, Corral \& Estévez, 2007; Gilbar \& Hevroni, 2007; Lisboa et al., 2002; Rodrigues \& Chaves, 2008; Silva, Muller \& Bonamigo, 2006; Smith, 2007). Essa incongruência pode ser considerada uma limitação do estudo conduzido, já que dificulta a comparação com os achados de outras pesquisas. Além disso, é importante que sejam considerados o nível de desenvolvimento cognitivo e os fatores demográficos, pessoais, socioculturais e ambientais, já que tais aspectos influenciam a avaliação da situação estressante e, consequentemente, a escolha pela estratégia de coping a ser utilizada (Peterson, 1989; Piko, 2001).

Quando comparadas as estratégias de coping mais usadas entre os sexos, verificou-se que a única estratégia que apresentou relevância estatística foi a de afastamento, que inclui esforços cognitivos de desprendimento e minimização da situação, sendo mais utilizada pelos meninos do que pelas meninas. Nos outros fatores não foram observadas diferenças significativas, confirmando os achados de Dell'Aglio e Hutz (2002). Outro estudo, que avaliou estratégias de coping em idosos institucionalizados, também não encontrou diferenças significativas quanto ao sexo (Vivan, 2007). Entretanto, pesquisas anteriores encontraram diferenças potenciais relacionadas a sexo e estratégias de coping (Ayers, Sandler, West \& Roosa, 1996; Carson \& Bittner, 1994; Compas et al., 1988; Kliewer \& Sandler, 1993; Weisz, McCabe \& Denning, 1994). Os resultados do estudo feito por Mathews (1982) demonstraram que os meninos são mais impacientes, agressivos e hostis, e utilizam mais estratégias de luta e competição.

A partir da correlação entre estratégias de coping e sintomas de stress, os resultados revelaram que, entre os sujeitos que apresentaram mais sintomas de stress, as estratégias mais utilizadas foram: fuga e esquiva, suporte social, confronto e afastamento. No estudo feito por Câmara e Carlotto (2007), foi possível identificar que as estratégias de aproximação do problema contribuem para um índice mais elevado de bem-estar psicológico, tanto para meninas quanto para meninos. Entretanto, os resultados obtidos por Sideridis (2006), em um estudo cujo objetivo foi avaliar a associação entre coping, afeto e stress, mostraram que não houve associação entre um tipo de estratégia (focalizada na emoção ou focalizada no problema) e níveis de stress. Todavia, o autor considera que a interação entre os dois tipos de estratégias diminui significativamente os níveis de stress, permitindo concluir que usar mais de um tipo de estratégia de coping é mais adaptativo do que usar apenas um tipo de estratégia. 
De um modo geral, os resultados permitiram identificar que os adolescentes deste estudo, diante de eventos estressores, tenderam a empreender esforços de regulação dos próprios sentimentos e ações, utilizar estratégias para evitar ou escapar do problema e empregar esforços cognitivos de desprendimento e minimização da situação, sendo esta última estratégia a única que apresentou diferença estatística significativa entre meninos e meninas, permitindo inferir que, de uma maneira geral, não há diferença entre os sexos (Folkman \& Lazarus, 1980). No entanto, meninos tendem a utilizar a estratégia de afastamento mais do que as meninas, talvez pelo fato de serem socializados de formas diferentes (Câmara \& Carlotto, 2007; Dell'Aglio, 2003).

Além disso, entre os adolescentes que apresentaram mais sintomas de stress, pode-se perceber que a maneira como eles lidam com situações estressoras pode estar relacionada ao alto nível de stress apresentado por eles, na medida em que não utilizam, frequentemente, estratégias de autocontrole, resolução de problemas e reavaliação positiva. Também se identificou que, nos indivíduos que apresentaram mais sintomas de stress, a busca por suporte informativo, tangível e emocional foi maior do que entre os sujeitos que apresentaram menos sintomas de stress. Essa busca por suporte social pode ser explicada pela dificuldade destes indivíduos em resolverem sozinhos situações de conflito, necessitando de ajuda externa. Outra hipótese é que pessoas com mais sintomas de stress estão mais expostas a situações avaliadas como estressoras, ou seja, apresentam uma demanda maior para a busca de auxílio.

\section{Considerações Finais}

As exigências da sociedade moderna trazem impacto significativo para a vida das pessoas que, cada vez mais, têm que se adaptar a situações que, muitas vezes, não se sentem aptas a enfrentar. Assim, é crescente a preocupação com as consequências dos eventos estressores para a saúde mental do indivíduo, uma vez que se verifica um aumento do stress não só na fase adulta, mas também em períodos anteriores do desenvolvimento, sobretudo na adolescência. Nessa etapa da vida, o stress está relacionado a estressores específicos que incluem questões associadas à família e à escola, além de mudanças corporais típicas da puberdade e

28 doença ou morte de pessoas próximas.
Diante do crescente aumento do stress entre adolescentes, é necessário entender como eles lidam com as situações de conflito, ou seja, quais as estratégias de coping que utilizam diante de eventos de vida estressores. Assim, o objetivo principal deste estudo foi identificar estratégias de coping utilizadas por adolescentes diante de eventos estressores, bem como a manifestação de sintomas de stress entre os participantes.

Os resultados mostraram que as estratégias mais utilizadas foram autocontrole, afastamento e fuga e esquiva, e as estratégias menos utilizadas foram as de confronto. Entre os indivíduos que apresentaram mais sintomas de stress, as estratégias mais utilizadas foram fuga e esquiva, suporte social, confronto e afastamento.

Não há um consenso entre os autores com relação às variáveis que influenciam a escolha das estratégias de coping utilizadas. Entretanto, é fato que a adoção de mais de uma estratégia é mais eficaz do que o uso de apenas um tipo, visto que o indivíduo tem mais recursos para enfrentar a situação estressante. Além disso, o conhecimento das estratégias utilizadas pelo indivíduo possibilita que este tenha mais flexibilidade para avaliar a eficácia delas. Consequentemente, ao perceber a ineficácia de determinada estratégia, o indivíduo terá melhores condições de escolher outra mais adequada para lidar com determinada situação estressante, evitando, assim, o stress.

A revisão da literatura sobre essa temática evidencia a escassez de estudos sobre coping no Brasil, considerando a importância das estratégias de enfrentamento para o bem-estar psicológico dos indivíduos. Destaca-se a necessidade de mais pesquisas que identifiquem estratégias de coping entre adolescentes, já que estudos anteriores avaliaram estratégias utilizadas diante de eventos estressores específicos, dificultando a comparação dos achados.

O entendimento aprofundado sobre o processo de coping pode colaborar para a implementação de programas que auxiliem na prevenção do stress e de outras psicopatologias. Dessa maneira, estudos como este são relevantes, já que incentivam os alunos a transformarem as estratégias utilizadas de forma automática em processos controlados, trazendo à consciência pensamentos e ações até então não conscientes. A partir do conhecimento da estratégia utilizada, o indivíduo é capaz de adequar suas ações às suas necessidades, 
encontrando soluções mais efetivas e mudando de estratégia quando esta não estiver funcionando ou quando não for adaptativa para o contexto.

\section{Referências}

Antoniazzi, A. S., Dell'Aglio, D. D., \& Bandeira, D. R. (1998). O conceito de coping: uma revisão teórica. Estudos de Psicologia (Natal), 3 (2), 273-294.

Ayers, T. S., Sandler, I. N., West, S.G., \& Roosa, M. W. (1996). A disposicional and situational assessment of children's coping: testing alternative models of coping. Journal of Personality, 64 (4), 923-958.

Becker, D. (1994). O que é a adolescência? (13a. ed.). São Paulo: Brasiliense.

Bussey, K., \& Bandura, A. (1999). Social cognitive theory of gender development and differentiation. Psychological Review, 106 (4), 676-713.

Byrne, D.G., Davenporta, S.C., \& Mazanov, J. (2007). Profiles of adolescent stress: the development of the adolescent stress questionnaire (ASQ). Journal of Adolescence, 30 (4), 393-416.

Calsbeek, H., Rijken, M., Bekkers, M. J. T. M., Henegouwen, G. P. V. B., \& Dekker, J. (2006). Coping in adolescents and young adults with chronic digestive disorders: impact on school and leisure activities. Psychology \& Health, 21 (4), 447-462.

Calvete, E., Corral, S., \& Estévez, A. (2007). Cognitive and coping mechanisms in the interplay between intimate partner violence and depression. Anxiety, Stress \& Coping, an International Journal, 20 (4), 369-382.

Câmara, S. G., \& Carlotto, M. S. (2007). Coping e gênero em adolescentes. Psicologia em Estudo, 12 (1), 87-93.

Carson, D. K., \& Bittner, M. T. (1994). Temperament and school-aged children's coping abilities and responses to stress. The Journal of Genetic Psychology, 155 (3), 289-302.

Castro, L. R. (1998). Infânciae adolescência na cultura demassa. Rio de Janeiro: Nau.

Compas, B. E. (1987). Coping with stress during childhood and adolescence. Psychological Bulletin, 101 (3), 393-403.

Compas, B. E, Banez, G. A., Malcarne, V. L., \& Worsham, N. (1991). Perceived control and coping with stress: a developmental perspective. Journal of Social Issues, 47 (4), 23-34.

Compas, B. E., Malcarne, V. L., \& Fondacaro, K. M. (1988). Coping with stressful events in older children and young adolescents. Journal of Consulting and Clinical Psychology, 56 (3), 405-411.

Dell'Aglio, D. D. (2003). O processo de coping em crianças e adolescentes: adaptação e desenvolvimento. Temas em Psicologia, 11 (1), 38-45.

Dell'Aglio, D. D., \& Hutz, C. S. (2002). Estratégias de coping de crianças e adolescentes em eventos estressantes com pares e com adultos. Psicologia USP, 13 (2), 203-225.
Estatuto da Criança e do Adolescente. (2007). Lei Federal 8.069, de 13.07.90. Porto Alegre: CMDCA.

Folkman, S., \& Lazarus, R. S. (1980). An analysis of coping in a middle-aged community sample. Journal of Health and Social Behavior, 21, 219-239.

Gazzaniga, M. S., \&Heatherton, T.F. (2005). Ciência psicológica: mente, cérebro e comportamento. Porto Alegre: Artes Médicas.

Gerrig, R. J., \& Zimbardo, P. G. (2005). A psicologia e a vida (16a. ed.). Porto Alegre: Artmed.

Gilbar, O., \& Hevroni, A. (2007). Counterfactuals, coping strategies and psychological distress among breast cancer patients. Anxiety, Stress \& Coping, 20 (4), 383-392.

Kaefer, H. (2006). Semiologia psicológica. In N. T. Rotta, L. Ohlweiler \& R. S. Riesgo (Eds.), Transtornos da aprendizagem: abordagem neurobiológica e multidisciplinar (pp.87-102). Porto Alegre: Artes Médicas.

Kaplan, H. I., Sadock, B. J., \& Grebb, J. A. (1997). Compêndio de psiquiatria: ciências do comportamento e psiquiatria clínica. Porto Alegre: Artmed.

Kliewer, W., \& Sandler, I. N. (1993). Social competence and coping among children of divorce. American Journal of Orthopsyquiatric, 63, 432-440.

Kristensen, C. H., Leon, J. S., D'Incao, D. B., \& Dell'Aglio, D. D. (2004). Análise da freqüência e do impacto de eventos estressores em uma amostra de adolescentes. Interação, $8(1), 45-55$

Lazarus, R. S., \& Folkman, S. (1984). Stress, appraisal, and coping. New York: Springer.

Levi, G., \& Schimitt, J. C. (1996). História dos jovens: da antigüidade à era moderna. São Paulo: Companhia das Letras.

Lipp, M. E. N. (1989). O tratamento do stress infantil. In E. F. M. Silvares (Org.), Estudos de caso em psicologia clínica comportamental infantil. Campinas: Papirus.

Lipp, M. E. N., \& Lucarelli, M. D. M. (1998). Manual da escala de stress infantil - ESI. São Paulo: Casa do Psicólogo.

Lipp, M. E. N., \& Novaes, L. (2000). O stress. São Paulo: Contexto.

Lisboa, C., Koller, S. H., Ribas, F. F., Bitencourt, K., Oliveira, L., Porciuncula, L. P., et al. (2002). Estratégias de coping de crianças vítimas e não vítimas de violência doméstica. Psicologia: Reflexão e Crítica, 15 (2), 345-362.

Losoya, S., Eisenberg, N., \& Fabes, R. A. (1998). Developmental issues in the study of coping. International Journal of Behavioral Development, 22, 287-313.

Mathews, K. A. (1982). Psychological perspectives on the Type A behavior pattern. Psychological Bulletin, 91 (2), 293-323.

Organização Mundial da Saúde. (2005). Relatório sobre saúde mental no mundo. Brasília: OMS.

Osório, L. C. (1992). Adolescente hoje. Porto Alegre: Artes Médicas.

Outeiral, J. O. (1994). Adolescer: estudos sobre a adolescência. Porto Alegre: Artes Médicas. 
Palacios, J. (1995). O que é a adolescência. In C. Coll, J. Palácios \& A. Marchesi (Orgs.), Desenvolvimento psicológico e educação: psicologia evolutiva (pp. 263-272). Porto Alegre: Artes Médicas.

Peterson, L. (1989). Coping with children undergoing stressful medical procedures: some conceptual, methodological, and therapeutic issues. Journal of Consulting and Clinical Psychology, 57 (3), 380-387.

Piko, B. (2001). Gender differences and similarities in adolescents' ways of coping. The Psychological Record, 51 (12), 223-235.

Raimundo, R. C. P., \& Pinto, M. A. P. M. (2006). Stress e estratégias de coping em crianças e adolescentes em contexto escolar. Aletheia, 24, 9-19.

Rodrigues, A. B., \& Chaves, E. C. (2008). Stressing factors and coping strategies used by oncology nurses. Revista Latino-Americana de Enfermagem, 16 (1), 24-28.

Romano, J. (1997). Stress and coping: a qualitative study of 4th and 5th graders. Elementary School Guidance \& Counseling, 31, 273-283.

Rotta, N. T. (2006). Dificuldades para a aprendizagem. In N. T. Rotta, L. Ohlweiler \& R. S. Riesgo (Orgs.), Transtornos da aprendizagem:abordagem neurobiológica emultidisciplinar (pp.113-123). Porto Alegre: Artes Médicas.

Ryan-Wenger, N. A. (1992). A taxonomy of children's coping strategies: a step toward theory development. American Journal of Orthopsychiatry, 62 (2), 256-263.

Ryan-Wenger, N. A., Sharrer, V. W., \& Campbell, K. K. (2005). Changes in children's stressors over the past 30 years. Pediatric Nursing, 31, 282-291.

Savóia, M. G., Santana, P., \& Mejias, N. P. (1996). Adaptação do inventário de estratégias de coping de Folkman e Lazarus para o português. Psicologia USP, 7 (1-2), 183-201.
Sideridis, G. D. (2006). Coping is not an 'either' 'or': the interaction of coping strategies in regulation affect, arousal and performance. Stress and Health, 22, 315-327.

Silva, J. D., Muller, M. C., \& Bonamigo, R. R. (2006). Estratégias de coping e níveis de estresse em pacientes portadores de psoríase. Anais Brasileiros de Dermatologia, 81 (2), 143-149.

Smith, C. S. (2007). Coping strategies of female victims of child abuse in treatment for substance abuse relapse: their advice to other women and healthcare professionals. Journal of Addictions Nursing, 18 (2), 75-80.

Sternberg, R. (2008). Psicologia cognitiva. Porto Alegre: Artes Médicas.

Taxis, J., Rew, L., Jackson, K., \& Kouzekanani, K. (2004). Protective resources and perceptions of stress in a multiethnic sample of school-age children. Pediatric Nursing, 30, 477-487.

Vivan, A. S. (2007). Estratégias de coping frente às dificuldades funcionais e sintomas depressivos em idosos institucionalizados. Dissertação de mestrado não-publicada, Pontifícia Universidade Católica do Rio Grande do Sul, Porto Alegre.

Weisz, J. R., McCabe, M., \& Denning, M. D. (1994). Primary and secondary control among children undergoing medical procedures: adjustment as a function of coping style. Journal of Consulting and Clinical Psychology, 62 (2), 324-332.

Recebido em: 19/9/2008

Versão final reapresentada em: 27/5/2009

Aprovado em: 26/6/2009 